EPJ Web of Conferences 45, 01132 (2013)

DOI: $10.1051 /$ epjconf/20134501132

(C) Owned by the authors, published by EDP Sciences, 2013

\title{
On the quadrant analysis of the flow in the street canyon
}

\author{
R. Kellnerová1, ${ }^{1,2, a}$, V. Fuka ${ }^{1}$, L. Kukačka ${ }^{1,2}$, V. Uruba ${ }^{2}$, and Z. Jaňour ${ }^{2}$
}

${ }^{1}$ Charles University in Prague, Faculty of Mathematics and Physics, The Department of Meteorology and Environment Protection, Czech Republic

2 Institute of Thermomechanics Academy of Sciences of the Czech Republic, v.v.i, Dolejškova 1402/5, Prague 182 00, Czech Republic

\begin{abstract}
It is well known that sweep and ejection are major contributors to the momentum flux in the turbulent boundary layer. In order to evaluate the contribution of these events to the total flux, the wind-tunnel data measured by PIV technique were analyzed from spatial point of view. It was revealed that both the sweep and the ejection reach up to $90 \%$ of the flux momentum inside the canyon. Moreover, the sweep and ejection pass the street in an alternative fashion. When sweep reaches maximum, the ejection falls down to minimum and vice versa. The TKE energy is also very high in these particular events, reaching 80-90\% of total TKE inside the street what makes the sweep and ejection to be the most important features in the flow.
\end{abstract}

\section{Introduction}

Research on coherent structures suggests that compact and organized features exist inside the turbulent flow. However, the complexity of the flow makes it difficult to reveal these patterns. In the recent decade, the PIV results substantially have helped to analyze flow dynamics. Unfortunately, there is a number of ways how to detect a coherent structures but there has not been draft a conclusion yet which one is both universal and reliable one. In this paper, the data from experiment are analyzes by Quadrant analysis.

The quadrant analysis deals with instantaneous values of momentum flux. Based on the Reynolds decomposition, the stress is divided into four categories after [1].

Since it is a decomposition from temporal point of view, it can be easily applied to one-point time-series of simultaneous measurement of two velocity components. From this reason, the method was frequently applied in the past.

We applied the Reynolds decomposition to a 2-D velocity field inside the street canyon obtained by PIV. The flow generated by street canyon is very turbulent and according to [2], certain quadrant events called sweep and ejection should be predominant contributors to the momentum flux.

\section{Experimental set-up}

The turbulent boundary layer is generated in the windchannel with a series of street canyons as a model. The canyons are identical, spanned from wall to wall and perpendicular to the approach wind (see Figure 1). Channel has $25 \mathrm{~cm}$ x $25 \mathrm{~cm}$ in cross-section and about $3 \mathrm{~m}$ in longitudinal direction. The area of interest lies behind the 21th row from the channel entrance, in order to provide a sufficient fetch for development of well-established internal

\footnotetext{
a e-mail: radka.kellnerova@it.cas.cz
}

boundary layer. Reference velocity measured on the channel axis at the mouth of the developing section is $5 \mathrm{~m} \cdot \mathrm{s}^{-1}$.

Two geometries of roof are used - triangle and flat shape. Scale in which the model is manufactured is 1:400. Therefore, $50 \mathrm{~mm}$ high and wide street corresponds to $20 \mathrm{~m}$ large street canyon in full scale (Figure 2). The street aspect ratio equals to one what determines a skimmingregime of the flow as concluded [3]. More detailed information about set-up could be found in [4].

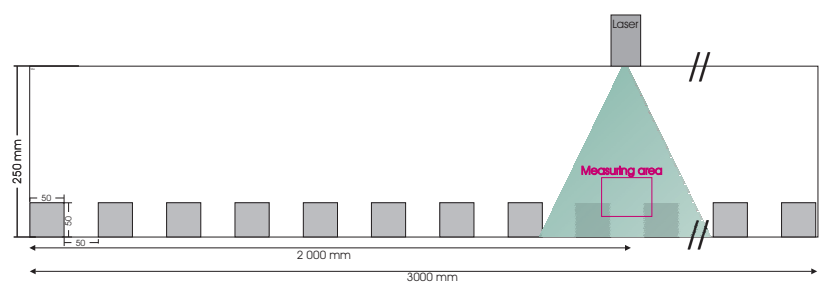

Fig. 1. Scheme of model of street canyons inside the channel. Flow is coming from the left side. Green area denotes the sheet light of laser for PIV.

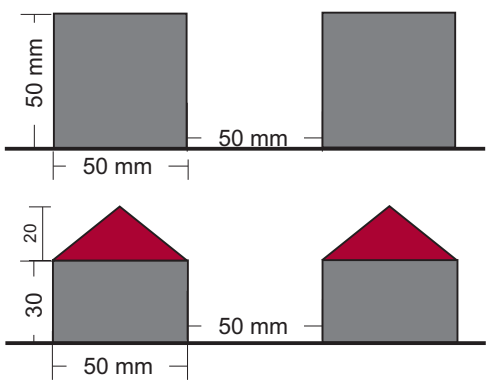

Fig. 2. Scheme of buildings with flat roof (upper) and pitched roof (lower). 
Firstly we verified if the measuring position is placed in the proper location. By CTA hot-wire anemometry, we measured a mean velocity and fluctuations. Gold-plated probe (Dantec 55 P01) with dimension of $5 \mu \mathrm{m} \times 1.25 \mathrm{~mm}$ measured vertical profile of longitudinal velocity with temporal resolution of $25 \mathrm{kHz}$. Acquisition time was $30 \mathrm{~s}$. The vertical profiles of mean velocity were taken in four consecutive canyons, the measurement position was placed in the inner one. The deviation from all four profiles does not exceed the $1.5 \%$. This confirms the boundary layer above the position reaches the equilibrium state with the surface beneath. Also, the lateral symmetry was checked.

Then, particle Image Velocimetry (PIV) with relatively high repetition rate $(500 \mathrm{~Hz})$ was installed. As the output from PIV system, we got 2-D vectors of instantaneous velocity in vertical plane. One run of PIV measurement consists of 1600 snapshots, each of them with more than 4800 velocity vectors. The spatial resolution turns out to be 1.2 $\mathrm{mm} \times 1.2 \mathrm{~mm}$ due to $50 \%$ of overlap. In following table, the parameters of PIV set-up are published.

\begin{aligned} & Diode pumped Nd:YLF laser \\ & \hline Repetition rate $500 \mathrm{~Hz} \\ &$ Repetition rate $1280 \times 1024 \mathrm{pxs} \\ &$ Interrogation area $32 \times 32 \mathrm{pxs} \\ &$ Overlapping $50 \%(80 \times 64$ vectors $) \\ &$ Energy $10 \mathrm{~mJ} \\ &$ Area $100 \times 100 \mathrm{~mm} \\ &$ Acquisition time $3.2 \mathrm{~s} \\ &$\hline\end{aligned}

Commercial software for PIV (DynamicStudio v3.00) was used to post-process of data. On the very beginning, for each ensemble of snapshots from one run of measurement, we subtracted an image mean from each individual images. Then we applied adaptive correlation, what is spatial correlation in specific interrogation area, and carried out the vector field. Term 'adaptive' herein refers to a dimension of interrogation area, which becomes smaller and smaller step-by-step until its final size is reached.

Several validation schemes filtered out the most striking spurious vectors and Moving average validation have replaced them by a reasonable estimation. Flow was filled by oily tracer particle with mean radius of $1 \mu \mathrm{m}$. Fortunately, due to a high level of turbulence, oil droplets were distributed evenly in the space. This allows the fast CCD camera to shoot a nice quality images with conveniently low number of wrong vectors.

After filtering and interpolating process, the time-series of wind speed in each location are extracted. The timemean momentum flux from the center of the canyon is plotted in Figure 3. For both building arrangements, the peak reaches the maximum approximately at roof-top level. In the pitched case, the peak is localized slightly higher and exhibits double value than in the flat case. Also, the vertical layer of enhanced flux is significantly wider in the pitched case. The vertical variation of pitched roofs apparently produces a higher level of turbulence intensity and the turbulence occupies a larger area - generates a wider shear layer.

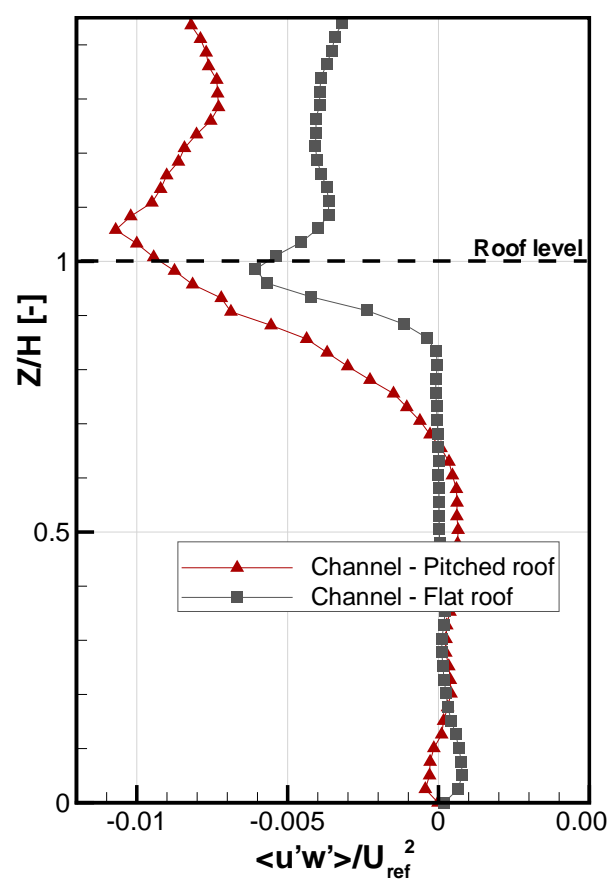

Fig. 3. Momentum flux in the center of street canyon with pitched and flat shape of roofs.

\subsection{Quadrant analysis}

The analysis is based on Reynolds decomposition, which decomposes the instantaneous velocity value into time-mean value $U$ and fluctuating term $u^{\prime}$. The momentum flux $u^{\prime} w^{\prime}$ is then divided into four quadrants according to scheme:

- $u^{\prime}>0, w^{\prime}>0 \quad 1$. quadrant - outward interaction

$\begin{array}{ll}-u^{\prime}>0, w^{\prime}<0 & \text { 2. quadrant - sweep }\end{array}$

- $u^{\prime}<0, w^{\prime}<0 \quad 3$. quadrant - inward interaction

- $u^{\prime}<0, w^{\prime}>0 \quad$ 4. quadrant - ejection.

The partial contribution from i-th quadrant to the total momentum flux $<u^{\prime} w^{\prime}>$ is obtained from formula of weighted average:

$$
\tau_{i}=\frac{<u^{\prime} w^{\prime}>_{i} \cdot N_{i}}{N_{\text {total }}}
$$

where $\left\langle u^{\prime} w^{\prime}\right\rangle_{i}$ means averaged flux within the i-th quadrant, $N_{i}$ is the number of events belonging to the i-th quadrant and $N_{\text {total }}$ is the global number of events recorded during time period .

Simply said, sweep transports the momentum from the faster free stream downwards. Considering the street canyon with vehicle traffic at the street level, sweep events brings relatively clear air from an up-roof area and then penetrates downwards into canyon. The ejection represents the slow, upward motion. In other words, ejection is responsible for transports of the heat, pollution or other scalar from the lower level upwards. These quadrants events strongly contribute to the so-called turbulent scalar fluxes ([5]).

The figure 4 shows the contributions to the total momentum flux from individual quadrants. The sweep and ejection are definitively the most dominant events in the turbulent flow. Especially in the locations where the turbulence increases. The other quadrants, inward and outward interactions, are almost negligible. 


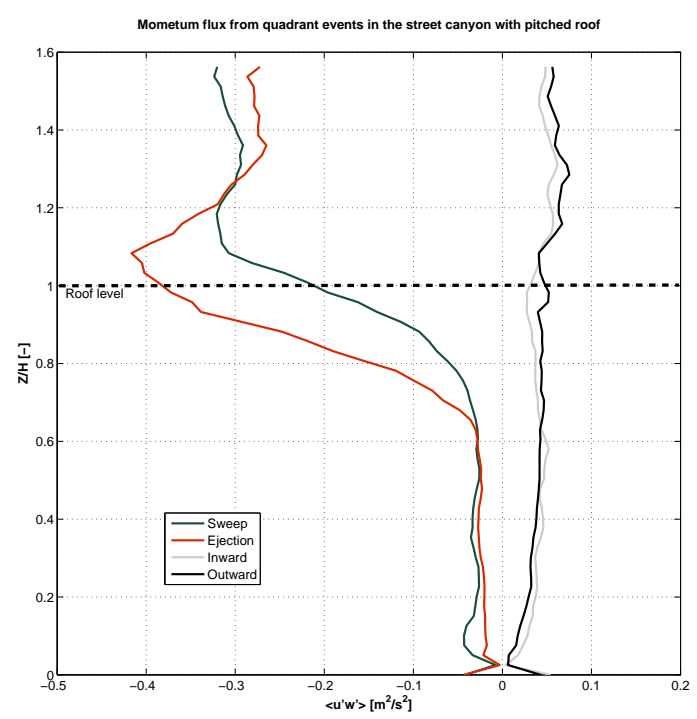

Fig. 4. Contribution from the particular quadrants to the momentum flux for pitched roof case.

Up to a certain elevation, the time-averaged sweep is consistently larger than the time-averaged ejection, however above this level, ejection dominates. We can also define a property $\delta S$ via substraction of the ejection from the sweep. $\delta S$ brings an information about prevailing momentum flux in the region.

$$
\delta S=\tau_{\text {sweep }}-\tau_{\text {ejection }} .
$$

If we evaluate the momentum flux belonging to the each quadrant separately and display its spatial distribution inside the street canyon, we will find out that only the results from sweep and ejection are interesting. The sweep and ejection events often travel in a compact shape across the street canyon. For better demonstration of this phenomena, it is convenient to display the difference $\delta S$. The following figures show the example of strong sweep (figures 5 - left) and ejection events (figures 6 - right). The negative (orange) values label the region where sweep is stronger than ejection whereas the positive (green) values symbolized the dominance of ejection.

The calculation of the relative spatially cumulative contribution from the event to the total momentum flux in every snapshot reveals, that sweep and ejection occasionally represent up to $80 \%-90 \%$ of the total momentum flux (see Figure 10). The negative value in Figure 10 of percentage is caused by negative sign of both the sweep and the ejection partial momentum flux. The inward and outward interaction have positive sign (check 4).

The before-mentioned conclusion is valid for both the arrangements - pitched and flat. This is surprising, because pitched roof produce much more violent flow. The ventilation inside the canyon is often disturbed, whereas the flat case generates relatively smooth flow where ventilation is much stable.

There is also clear tendency for both events to pass the street canyon in alternating fashion. Indeed, the mutual correlation coefficient between relative contributions of sweep and ejection is -0.9. If the sweep comes into canyon, the ejection is suppressed and vice versa.

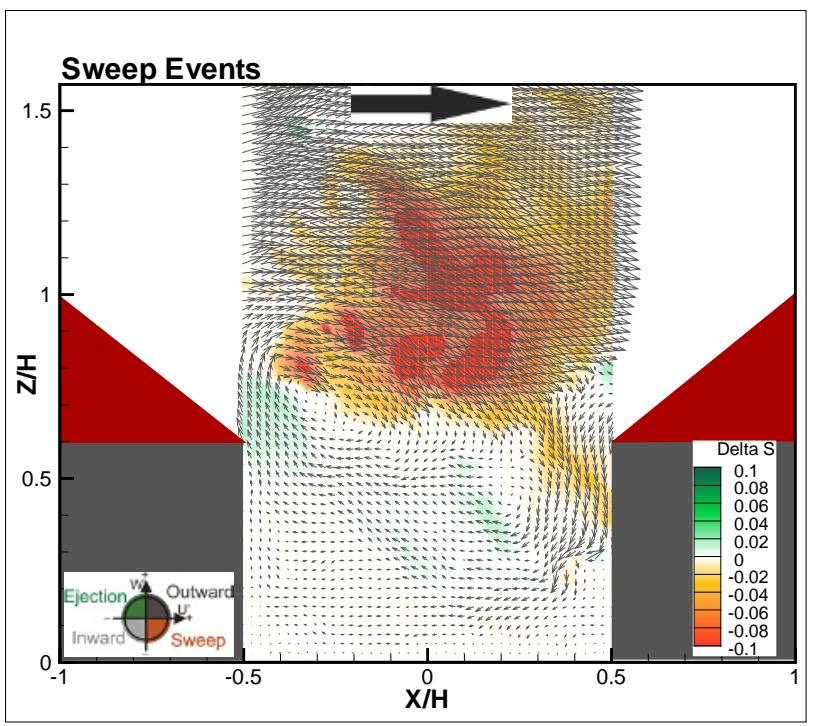

Fig. 5. The dominance of sweep fraction of the momentum flux.

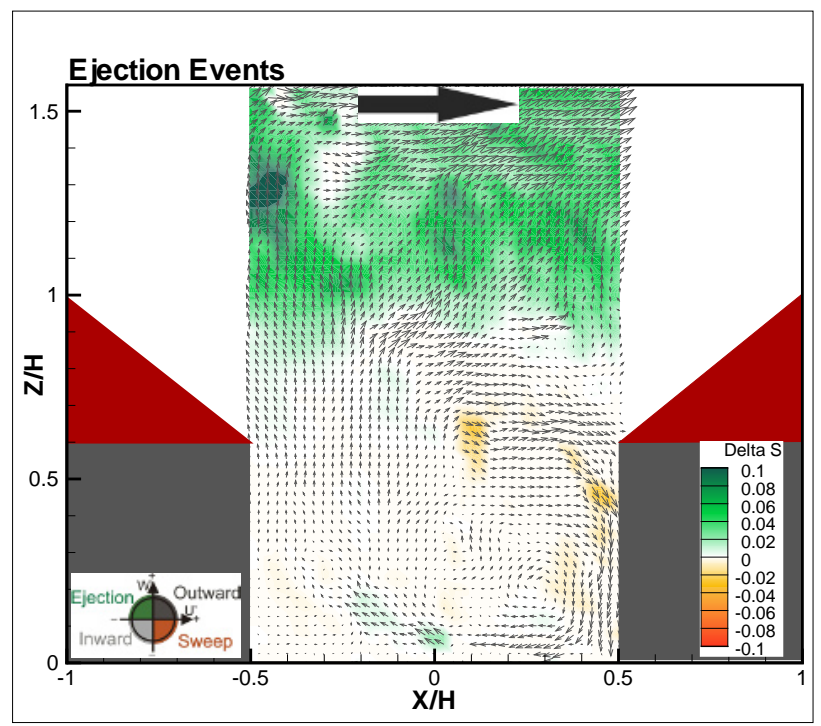

Fig. 6. The dominance of ejection fraction of the momentum flux.

Notwithstanding, the outward and inward interaction rarely contain $40 \%$ of momentum flux and do not exhibit any mutual correlation, their correlation coefficient is only -0.16 .

When defining threshold of $40 \%$ in relative contribution, which clearly determines the exclusivity of the sweep or ejection presence in the canyon, the intermittency factor, based on the definition in [6], is 0.4 for both the sweep and ejection (see table below). So together they cover $80 \%$ of the record time. If the prevailing wind in full scale reaches $5 \mathrm{~m} \cdot \mathrm{s}^{-1}$, these events would last typically for about $10 \mathrm{~s}$ and their scale would be around $30 \mathrm{~m}$. In the model scale it corresponds to $70-80 \mathrm{~mm}$ around $50 \mathrm{~mm}$ large buildings.

\begin{tabular}{lcccc} 
Threshold 40\% & Outward & Sweep & Inward & Ejection \\
\hline Intermittency & 0.01 & 0.40 & 0.01 & 0.39 \\
Life-time (full scale) [s] & 1 & 10 & 2 & 12 \\
\hline
\end{tabular}


With increasing the threshold, the less and less intermittency is obtained. In the flat case, the intermittency falls down a little bit more quickly. So, there is another small distinction between flow generated by flat and pitched roof geometries.

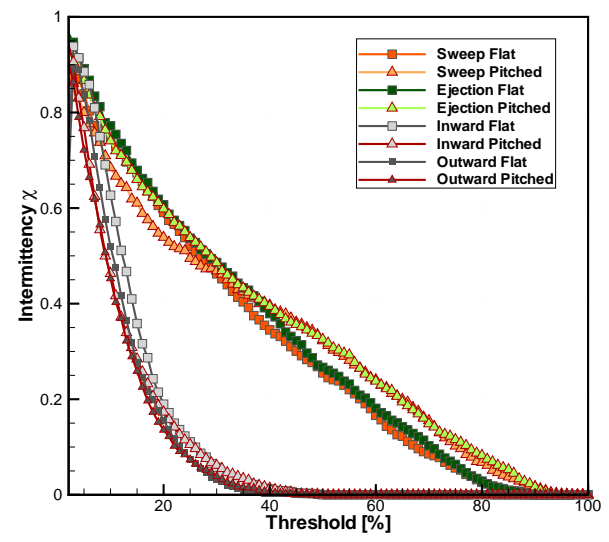

Fig. 7. Intermittency factor as a function of threshold in percentage of contribution to the total momentum flux from quadrant events.

From Figure 11 is apparent that most of the sweep and ejection happens above the roof. When returning back to the picture of momentum contribution, we added a thicker line representing the contribution coming from the region above the roof (Figure 11). Thin line stays for the previous total momentum flux in the whole canyon.

We can conclude that majority of these specific fluctuating motions, almost $90 \%$ of the flux, takes place above roof. The explanation is simple, the longitudinal velocity above the roof is much faster and so the fluctuations vary strongly in magnitude. Deeper, between the building walls, the flow strongly decelerates and velocity of fluctuations decreases as well in term of magnitude. Only the ratio of the fluctuation to the mean velocity significantly increases what causes a high value of turbulence intensity thanks to its definition in [7].

Regarding the turbulent kinetic energy, the sweep and ejection are extremely dominant, they contain $80-90 \%$ of total TKE in the canyon (Figure 12). Again, the percentage is the same for flat and pitched case.

However, the inspection of the typical picture of the flow dynamics between the roofs reveals that associating dynamical patterns are completely different. While only a small vortices travel almost exclusively at the roof-top level in the flat case (Figure 8), the deformation of the flow field and scale of the patterns themselves is much larger in the pitched case (Figure 9). We can draft a conclusion that bigger flow structures induce bigger interactions between the free stream and the street canyon. The vertical variability of the pitched roof acts in the same way as a group of buildings with various heights. Both built-up areas would generate a higher momentum and intensive scalar flux.

\section{Conclusions}

The Quadrant analysis was applied to the data from very turbulent flow inside the street canyon with two different

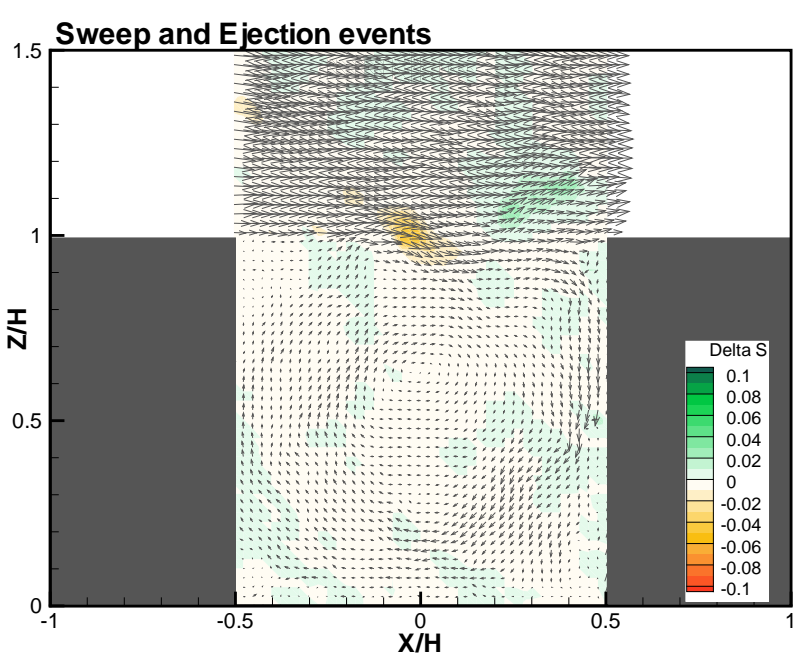

Fig. 8. Typical pattern in the flow over the flat roofs with emphasized sweep and ejection features.

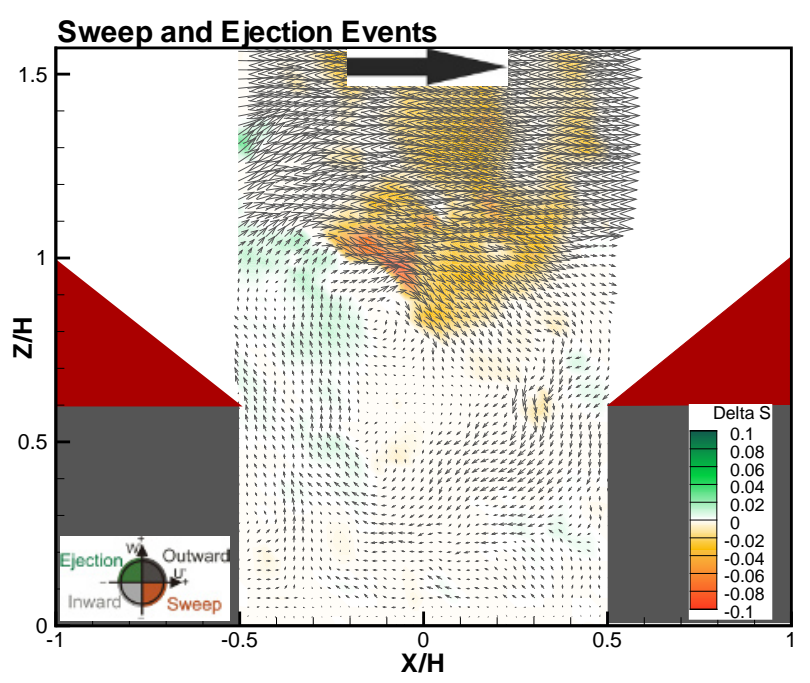

Fig. 9. Typical pattern in the flow over the pitched roofs with emphasized sweep and ejection features.

shapes of roof. It was confirmed that both the sweep and the ejection represent definitively the most important fractions of the momentum flux. When considering relative contribution to the total momentum flux inside the street from spatial point of view, both the sweep and ejection contribute by $80-90 \%$. Although, in absolute numbers, the peak of momentum flux localized at the pitched roof-top level is double than the one at flat roof-top level, the relative contribution is the same. The sweep and ejection also contain a majority of TKE - up to 80-90\%. However, the spatial extents of shear layers distinguish from each other and the structures of the feature are completely different.

\section{Acknowledgement}

The authors kindly thank the Czech Science Foundation GACR (project GAP101/12/1554) for their financial support. 


\section{References}

1. W.W. Willmarth, S.S. Lu, Journal of Fluid Mechanics, 55, 65 (1972)

2. W. Zhu, Ph.D. thesis, The Johns Hopkins University (2006)

3. T.R. Oke, Boundary Layer Climates (Routledge, London, 1987)

4. R. Kellnerova, L. Kukacka, K. Jurcakova, V. Uruba, Z. Janour, Journal of Wind Eengineering and Industrial Aerodynamics 104, 302 (2012)

5. L. Kukacka, S. Nosek, R. Kellnerova, K. Jurcakova, Z. Janour, Scientific World Journal (2012)

6. S. Pope, Turbulent Flows, (Cambridge University Press, New York, 2000)

7. VDI, Physical modelling of flow and dispersion processes in the atmospheric boundary layer - aplication of wind tunnels, Beuth Verlag, Berlin (2000)

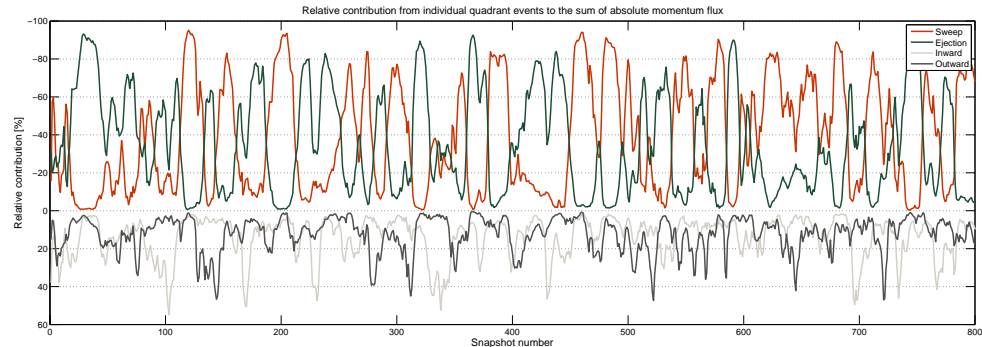

Fig. 10. The time evolution of the relative contributions to the absolute total momentum flux - pitched case.

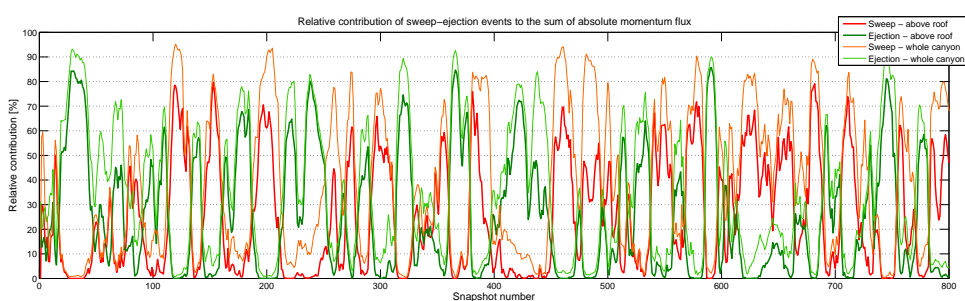

Fig. 11. The time evolution of the relative contributions from sweep and ejection in the region above the roof (thick line) and from the whole street canyon (thin line) - pitched case.

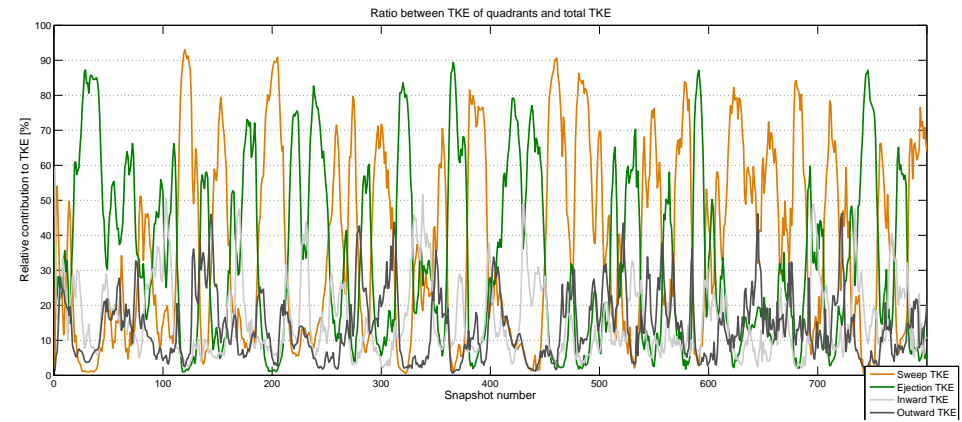

Fig. 12. The time evolution of the contributions from sweep and ejection to the total TKE - pitched case. 